\title{
A comparison of low cost wireless communication methods for remote control of grid-tied converters
}

\author{
Terashmila Lasagani K A., Tariq Iqbal, George Mann, \\ Faculty of Engineering and Applied Science, Memorial University of Newfoundland, Canada \\ St. John's, NL A1B3X
}

\begin{abstract}
Remote control of grid tied converters has become important with an increasing number of grid connected energy storage systems. Hence, it is crucial point for utilities to outline the requirements of the communication methods and to find the best communication infrastructure to handle the remotely located converters in a reliable and cost effective manner throughout the operation. The purpose of this study is to come up with a low cost wireless communication solution for remote controlled converters. Focusing on low cost, this letter studies about three communication methods which can be applicable to control the remote converters. All three methods are being tested to find out the suitability for data communication. Then this paper provides a comparison of all three methods with cost, measured range and the data rate. And conclude that LoRa is the solution with the highest potential for this application from the compared methods.
\end{abstract}

Keywords-SCADA, LoRa, Radio Teletype, VHF/ UHF data

\section{INTRODUCTION}

Due to challenges like growing population and increasing demand for energy, the global climate change, an increase in carbon emissions, energy storage problems, the capacity limitations of electricity generation, governments and utilities have been provoked to address these issues with a new approach of electricity generation. To address those issues renewable energy generators has emerged as a promising technology [1]. Recently, renewable energy has been spread in world in a level of solar systems with few kilo watts of generation up to some large scale solar and wind systems with some megawatts of generation. The integration of such renewable generators into the main grid is transforming the present power system in a large scale Distributed Generation (DG) system which is incorporating different technologies, voltage, currents and power levels and etc [1], [2], [3].

Therefore, due to increasing number of inverters connected to the main grid, the communication technologies have become essential to resize the envisioned grid in order to enhance the efficiency, reliability and safety, with smooth integration of renewable and alternative energy sources [4]. Also DG requires a two-way communication infrastructure in order to handle the remotely located inverters and to control them from a central station as per the demand requirements.
As per the literature survey, along with the new concept of smart gird several communication technologies have been employed in communication between inverters and central location. This central location can be a single computer serving as a server and SCADA system with sophisticated equipment depending on the energy source capacity.

Both wired and wireless media of communication technologies has been used for data transmission. Depending on the situation wireless communication methods shows some advantages upon wired technologies like low cost infrastructure and ease of connection to difficult or unreachable areas. As suggested in literature survey, communication between inverters and SCADA or server can be done via power line communication (PLC), optical fiber or wireless communications such as Zigbee, Cellular (3G), LTE (4G), Wi-Fi, Bluetooth and Radio Frequency (RF) [5].

However as per the study each of which method has their own pros and cons based on their deployment. Monthly operational cost of both Cellular (3G) and LTE (4G) and especially the low range of $3 \mathrm{G}$ technology can be presented as the limiting factors. Even though Zigbee is with relatively low power usage, less cost and less complexity, since it has low processing capability and small delay requirements, it cannot be considered as a fine selection for practical implementations.

The high cost and additional time requirement for building the infrastructure are the main constraints in wired technologies like optical fiber and PLC, but their ability in providing a high capacity, reliability and security is significant. [5]

The communication infrastructure between energy generation, transmission, and distribution and consumption requires twoway communications, inter-operability between advanced applications and end-to-end reliable and secure communications with low-latencies and sufficient bandwidth as per T. Sauter et. al. [6]. Also as per V.C. Gungor et al., following features can be listed as the main requirements of the communication method which should comprised with [5].
a. Signal availability,
b. Overall Cost
c. Security
d. Transmission Distance
e. System reliability and robustness 


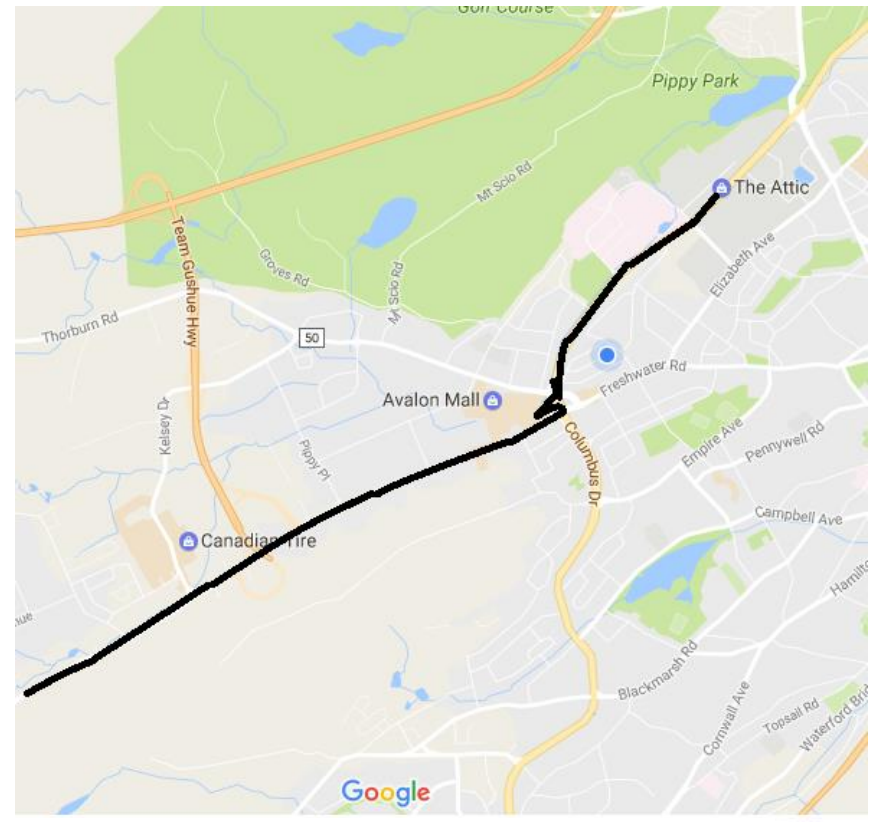

Fig. 1 Path travelled

The purpose of this study is to find out a low cost communication method which can be deploy in between inverter of the renewable energy source and server or SCADA. Therefore, in the current research we focus on three wireless low cost communication methods, namely LoRa, old Radio teletype technology and/VHFwireless data transmission modules. In the rest of the paper the experimental set up which used for testing those methods in suitability for data transmission, overview of each selected technology and the comparison with respect to cost, range and baud rate and the power consumption is presented.

\section{RANGE TESTING SETUP}

Three available technologies are tested to find out the maximum distance. The measurements took in St. John's, Newfoundland, Canada in 2016 December. One end kept stationary and other end moved. In order to give unbiased conditions to all options, the stationary end was same for each and same route was followed for all four technologies. Fig. 1

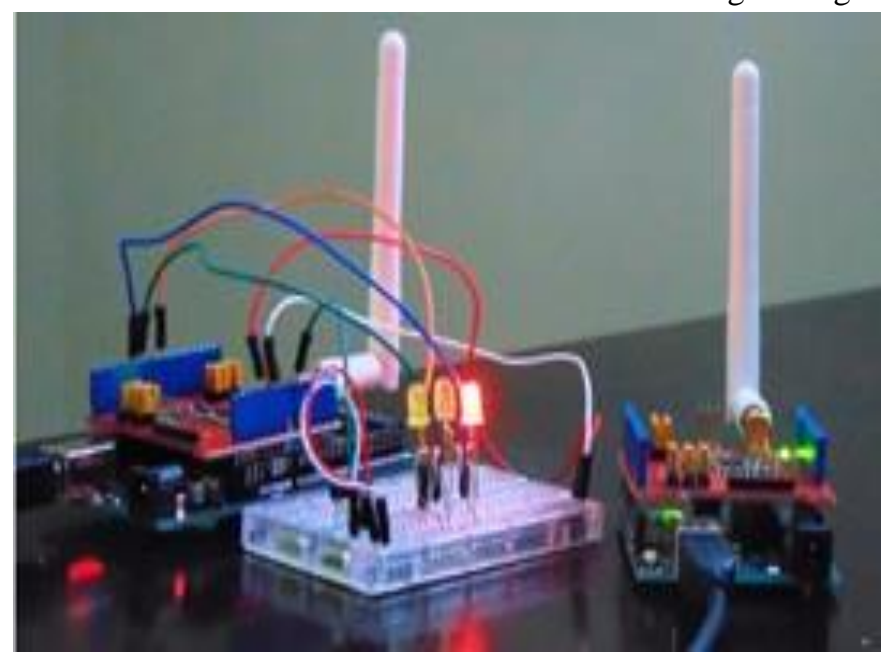

Fig. 2Dragino LoRa shield based setup shows path followed for testing. Point which is shown as "The Attic" was the base station. It is situated 7 meters above the ground level. Moving end was mounted on a car. IPhone was used in order to track the path from the stationary end.

\section{LORA}

There are four main communication technologies emerging for IoT based applications. Most of researches are being conducted to develop a cellular network for low power wide area network. Sigfox, LTE-M, NB-IoT develop their own mobile network for IoT [7]. The main drawback is that all these three comes with a periodical fee for the infrastructure like a mobile service provider. For example, Sigfox comes with an annual subscription fee and technology like Sigfox does not have enough coverage. LoRa is also developed for IoT based applications but it allows user to use own backbone network [8].

LoRa is a new spread-spectrum modulation technology which is named as LoRa use wideband linear frequency modulated chirp pulses for encoding data. In Direct Sequence Spread Spectrum (DSSS), carrier phase of transmitter changes with a code sequence which is generated by multiplying the data with a spread code. Chirp Spread Spectrum was used in Radar applications for military applications. Due to its many features such as low power requirement, inherent robustness, resistance to Doppler effect IEEE adopted this for Low-Rate Wireless Personal Area Networks (LR-WPANs) (standard 802.15.4) [9]. LoRa has the ability to communicate over large range. However, it communicates with very low data rate. Key features of the LoRa technology can be listed as below.

1. Large range of communication: LoRa has the ability to communicate over $22 \mathrm{~km}$ of distance with line-of-sight (LOS) and even without the LOS, it can communicate for a distance of more than $2 \mathrm{~km}$.

2. Low path loss: LoRa uses the $868 \mathrm{MHz}$ and $915 \mathrm{MHz}$ ISM bands for its communication unlike most of the other technologies uses $2.4 \mathrm{GHz}$ band for the communication. Therefore, with use of low frequency band in LoRa, it has achieved lower path loss than conventional wireless technologies.

3. Good sensitivity: In wireless communication, achieving a higher link budget is very useful for successful communication. LoRa has the ability to achieve around 148 $\mathrm{dB}$ link budget which implies LoRa is capable to handle a

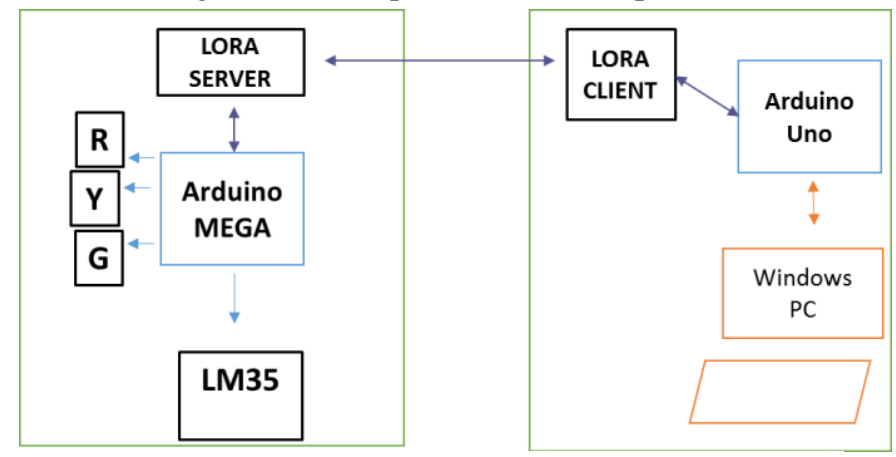

Fig. 3 Block diagram for Dragino LoRa shield based setup 


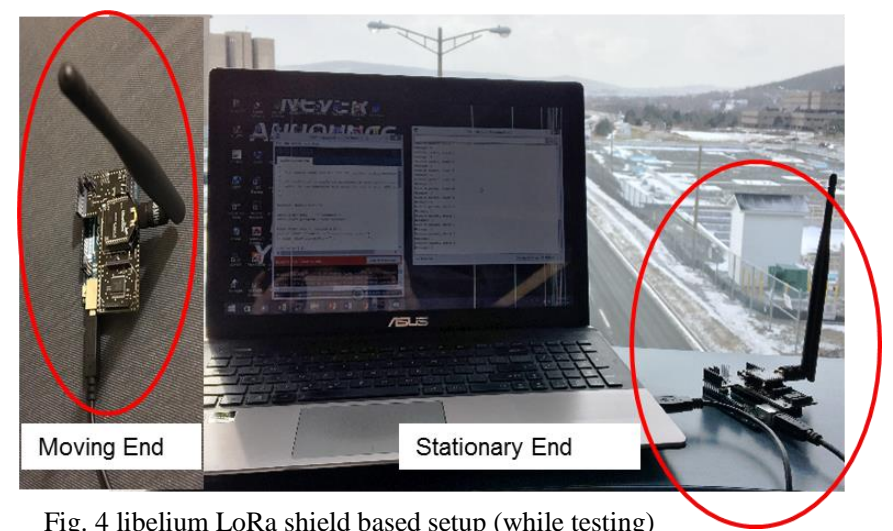

Fig. 4 libelium LoRa shield based setup (while testing)

successful communication.

4. Fewer Interference: Since LoRa uses $868 \mathrm{MHz}$ and 915 $\mathrm{MHz}$ ISM bands which is not as popular as $2.4 \mathrm{GHZ}$ band in the communication, it has lower inferences than the popular bands. Lower the inferences mean higher the signal quality and reliability.

5. Good obstacle penetration: Since LoRa uses lower frequency than other communication methods, it has higher ability to penetrate and that makes LoRa is more suitable to use in urban environment.

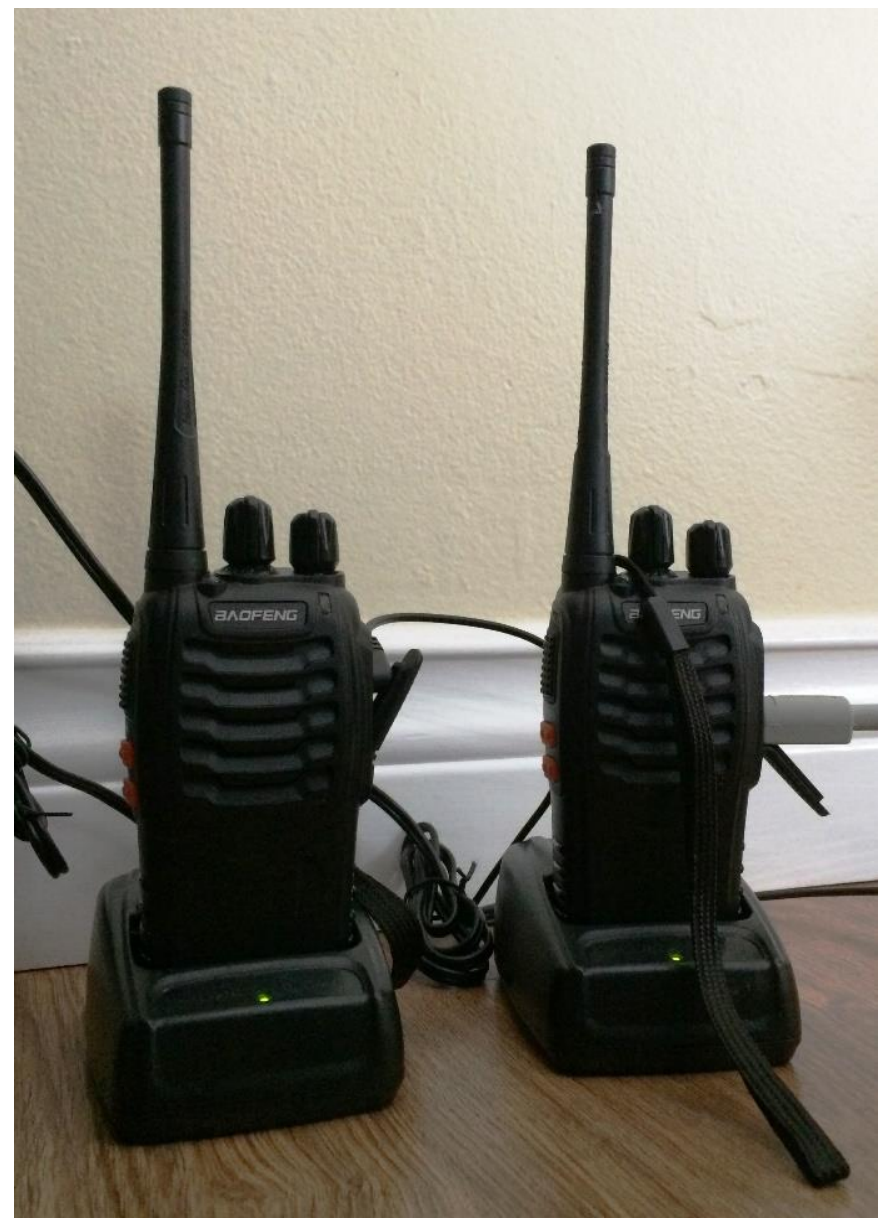

Fig. 6 One identical end of the RTTY based system

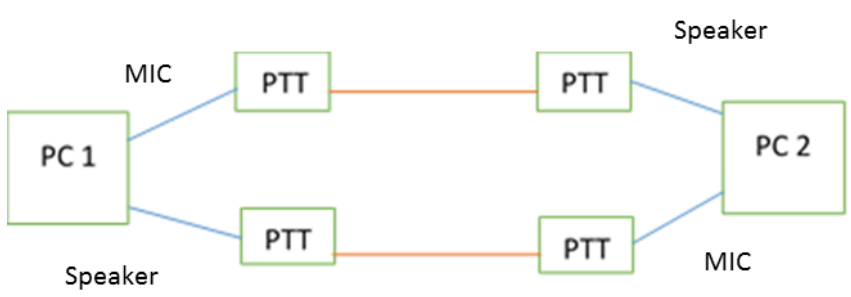

Fig. 5 Block diagram for RTTY based setup

\section{A. Dragino LoRa Shield}

Fig. 2 shows Dragino LoRa Shield that is a low cost shield. Two units cost only CAD 55.00 but range wise it only gives up to $220 \mathrm{~m}$ as per our measurements. In order to test, experimental setup has been built based on the block diagram shown in the fig. 3. Where the LORA client kept stationary while the server moving on the path shown in fig. 1. Arduino Mega was used in the server side, while Arduino Uno was used in the client side.

In this setup LM35 measured the temperature and sent it per each second to the client at $9600 \mathrm{bps}$ through the LORA link. From the code the system has been implemented as a full duplex link where the client sent an acknowledgement or a command to the server to operate.

\section{B. LibeliumLoRa Shield}

And the other LORA kit produced and distributed by Libelium shown in fig. 4. It costs about CAD250.00. The measured range is $4.5 \mathrm{~km}$. And it has more embedded features than the Dragino shield. It provides 13 channels to the user which allows user to develop own cellular network. It also allows user to control the transmitting power and this feature is important to design the cellular network and determining cell size, cell radius and etc.

Since the objective of this test to obtain the maximum distance, the nodes were configured to use the longest distance by maximizing the output power to $25 \mathrm{~mW}$. In order to test the range these modules' moving end programmed to send a number sequence for each four seconds at $9600 \mathrm{bps}$. And the receiving end was programmed to send received number to the PC for observations. Fig 4 shows a picture of the stationary side while testing. For both ends Arduino Uno boards were used.

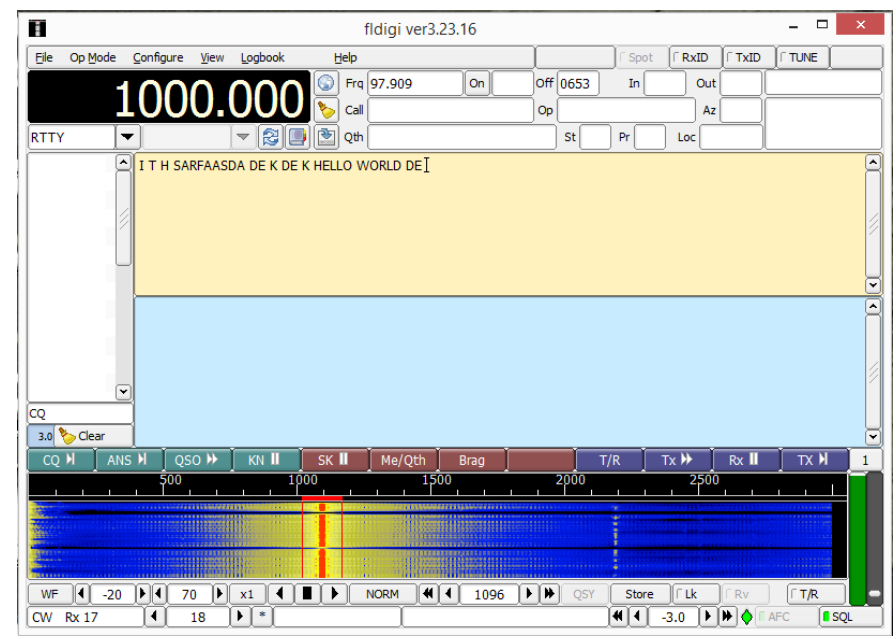

Fig. 7 FLDIGI interface during tests in progress. 
$\$ \mathrm{a}=1$

$\$$ port $=$ new-Object System. I0.Ports.SerialPort CoM9, 9600, None, 8 , one sport.open ()

DO

\{

"Starting Loop \$a"

$\$$ port. WriteLine $(\$ a)$

$\$ a++$

"Now " $\$ a$ is $\$ a "$

\} While (1)

Fig. 8: PowerShell script

\section{RADIO TELETYPE}

Traditional Land Mobile Radio (LMR) networks are being using for voice communications. But over decades same LMR devices used for data communications as well [10]. Before internet coming to the arena hobbyist used this Radio Teletype technology for keyboard to keyboard communications. In this approach two sets of half duplex ham radios made by BAOFENG are being used for data communication. These are $5 \mathrm{~W}$ radios that uses $400-470 \mathrm{MHz}$ band for communications. Software called FLDIGI is used as the interface. There are number of open source RTTY software which can be found on the internet such as MMTTY. Fig. 5 shows the block diagram of the system and Fig. 6 shows one end of the setup and other end will be identical. As shown in Fig. 5 and 6, this approach is simple. Two computers with FLDIGI installed have been used as the client and the server side [11]. FLDIGI can be used for RTTY communications. This software is mainly used by Amateur radio operators. Microphone and the speaker of the ham radio are connected to computer's headphone and microphone connections. In order to test the range one end kept stationary while other end moving on the path specified in the figure 1 . And it gave $7 \mathrm{~km}$ range during the measurements. Fig. 7 shows the FLDIGI interface during the testing.

\section{VHF/UHF WIRELESS DATA MODEM}

From the middle of the last century, there are number of companies who develop these data modems. For testing and verifications in this research, two set of data radios made by Lensen are being used. Main advantage of this is that both are easy to use where the user can simply send data using serial port [12].

In order to test this approach PowerShell script shown in fig. 8 has been used. It will generate a number from the nonmoving end. And the moving end monitor for that number. During testing, in a case of moving end unable to receive desired number that end had been stopped moving and the antenna was taken out of the vehicle. Since the objective of this research is to measure the range lowest baud rate has been used which is $1200 \mathrm{bps}$.

\section{A. $1 W$ wireless data module}

LS-U1000 is $1 \mathrm{~W}$ wireless data module smaller in size. The only difference is that the second one is $25 \mathrm{~W}$. Communication link is around 120.00 CAD. This $1 \mathrm{~W}$ module can be powered with USB power even through the PC. And it has 1200, 2400, $4800,9600,19200 \mathrm{bps}$ baud rates that can be chosen as per the requirement. Main drawback with this system is that these are half duplex. In order to make full duplex, time division multiplexing has to be done through the program. Fig. 9 shows both ends of the communication link.

\section{B. $25 W$ wireless data module}

LS-V25000 is $25 \mathrm{~W}$ and separate power supply is needed to supply power to the module. And it uses MSK modulation (Minimum-shift keying) and 1200, 2400bps baud rates. And two ends of this communication are shown in the fig. 11.

\section{COMPARISON}

In order to find out the best communication method, all available technologies are compared with respect to cost, power consumption, measured range and data rate and tabulated in the Table I.

As shown in the Table I, Dragino LoRa Shield has only $220 \mathrm{~m}$ measured range while all other technologies have more than $2 \mathrm{~km}$ range while testing. But power consumption of that module is the second lowest among all but if per $\mathrm{km}$ power

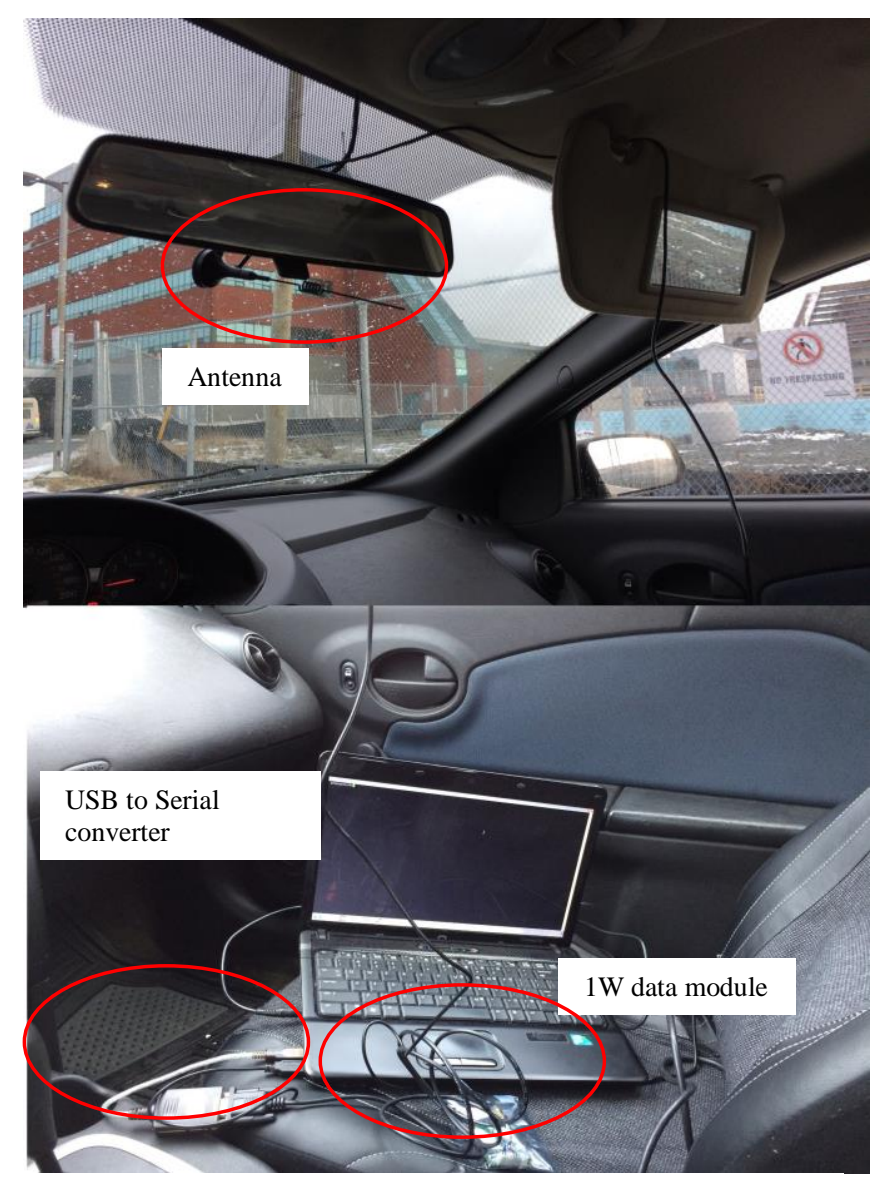

Fig. 9: Testing 1W wireless module 
consumption considered that will be about $1 \mathrm{~W} / \mathrm{km}$. Due to distance restriction Dragino LoRa Shield has to be omitted. Plus, unlike libelium LORA this doesn't divide the spectrum in to channels. TDM and TDMA need to use for a population of inverters.

When ham radio option is considered, it has the second highest distance among them all. But data rate wise it has a very low baud rate. Plus, this uses common band which is being used for many applications which results high interferences and low security. Due to those issues radio teletype technology has also been rejected. But this can be combined with an another option which is half duplex like the $1 \mathrm{~W}$ VHF/UHF data modem.

$1 \mathrm{~W}$ can be considered as a good option because it has a very good power per $\mathrm{km}$ factor which is about $0.303 \mathrm{~W} / \mathrm{km}$. But this module is half duplex. There is no way to use two modules parallel to each other with frequency multiplexing because this doesn't provide any channels. Therefore, only option is to use time division multiplexing to make it full duplex. It is also possible to implement. But when it comes to use these for more than one inverter where in a population of inverters it will be hard to provide multiple access. With TDMA this can be improved up to limit. Because when the number of users increased it will be difficult to provide real time data and real time controlling for the unit. And main drawbacks in TDMA like synchronization problems will make this task more difficult.

Libelium LORA module and $25 \mathrm{~W}$ wireless modem are the two options left. Depends upon the distance requirement one of these can be selected. Libelium LORA module provides 13 channels. This allows user to use FDM and it also allows user to improve the network with FDMA. If user needs to improve the number of inverters in the population user will be able to use Hybrid FDMA/ TDMA method. Therefore, with using this technology a LORA network can be designed for a large population of inverters distributed across a wide area based on cellular architecture. And the other advantage which Lebelium LORA having is that it allows user to control power that will enables user to control the size of the cell as well.

\begin{tabular}{|l|l|l|l|l|}
\hline & $\begin{array}{l}\text { Cost } \\
\text { (CAD in } \\
\text { 2016) }\end{array}$ & $\begin{array}{l}\text { Power } \\
\text { Consumption } \\
\text { (transmitting) }\end{array}$ & $\begin{array}{l}\text { Measured } \\
\text { Range }\end{array}$ & $\begin{array}{l}\text { Data rate } \\
\text { (bps) } \\
\text { Baud rate }\end{array}$ \\
\hline $\begin{array}{l}\text { Dragino } \\
\text { LoRa } \\
\text { Shield }\end{array}$ & 55.00 & $100 \mathrm{~mW}$ & $220 \mathrm{~m}$ & $\begin{array}{l}30000 \\
(\mathrm{max})\end{array}$ \\
\hline $\begin{array}{l}\text { Libelium } \\
\text { LORA } \\
\text { shiled }\end{array}$ & 250.00 & $25 \mathrm{~mW}$ & $4.5 \mathrm{~km}$ & $980-5470$ \\
\hline $\begin{array}{l}\text { 4 Ham } \\
\text { radios }\end{array}$ & 140.00 & $5 \mathrm{~W}$ & $7.2 \mathrm{~km}$ & 50 \\
\hline $\begin{array}{l}\text { 1W data } \\
\text { module }\end{array}$ & 120.00 & $1 \mathrm{~W}$ & $3.3 \mathrm{~km}$ & $\begin{array}{l}1200,2400 \\
, 4800 \\
, 9600 \\
, 19200\end{array}$ \\
\hline
\end{tabular}

\begin{tabular}{|l|l|l|l|l|}
\hline $\begin{array}{l}\text { 25W data } \\
\text { module }\end{array}$ & 600.00 & $25 \mathrm{~W}$ & $2.0 \mathrm{~km}$ & 1200,2400 \\
\hline
\end{tabular}

\section{CONCLUSION AND FUTURE WORKS}

Main objective of this research is to find out a communication method for a SCADA where equipment is distributed away from the central processor around few kilometers. Data gathered are needed to be sent to the central processor for data processing. Available technologies are documented through a detailed literature survey. Three free band based technologies, LORA, radio teletype technology and VHF/UHF data modules are being tested. In the comparison all three approaches are compared with respect to cost, measured range and the data rate. After comparing features Libelium LORA module and $25 \mathrm{~W}$ wireless modem left as only options and one of that can be selected according to the distance requirement.

For further works, directional antenna will be added to the LORA module to improve the range and also a software will be developed to use communication method to access SCADA system remotely using a low cost client.

\section{ACKNOWLEDGMENT}

This project is being funded by NSERC Energy Storage Technology (NEST) Network. And our special thanks goes to WEIcan for their support.

\section{REFERENCES}

[1] H. Kanchev, D. Lu, F. Colas, V. Lazarov and B. Francois,. 2011 Oct. "Energy Management and Operational Planning of a Microgrid With a PV-Based Active Generator for Smart Grid Applications." IEEE Transactions on Industrial Electronics 4583-4592.

[2] P. Palensky, D. Dietrich. 2011. "Demand side management: Demand response, intelligent energy systems, and smart loads,." IEEE Trans. Ind. Inf. 7 (3): 381-388.

[3] Q.Yang, J. A. Barria, and T. C. Green,. 2011. "Communication infrastructures for distributed control of power distribution networks." IEEE Trans. Ind. Inform. 316-327.

[4] V. C. Gungor, B. Lu, G. P. Hancke,. 2010 . "Opportunities and challenges of wireless sensor networks in smart grid." IEEE Trans. Ind. Electron 57 (10): 3557-3564.

[5] V. C. Gungor et al. 2011. "Smart Grid Technologies: Communication Technologies and Standards." IEEE Transactions on Industrial Informatics 529-539.

[6] T. Sauter, M. Lobashov,. 2011. "End-to-end communication architecture for smart grids." IEEE Transactions on Industrial Informatics 529-539.

[7] J. Petajajarvi, K. Mikhaylov, A. Roivainen, T. Hanninen and M. Pettissalo,. 2015. "On the coverage of LPWANs: range evaluation and channel attenuation model for LoRa technology." 2015 14th International Conference on ITS Telecommunications (ITST). Copenhagen. 55-59.

[8] n.d. What is LORA? SEMTECH. http://www.semtech.com/wirelessrf/internet-of-things/what-is-lora/.

[9] Corporation, Semtech. 2015. AN1200.22 LoRa ${ }^{\mathrm{TM}}$ Modulation Basics.

[10] G. Eason, B. Noble, and I.N. Sneddon, "On certain integrals of Lipschitz-Hankel type involving products of Bessel functions," Phil. Trans. Roy. Soc. London, vol. A247, pp. 529-551, April 1955.

[11] Cass, Stephen. 2016. A ham radio for makers [Resources_Hands On]. IEEE Spectrum, 21-23.

[12] n.d. http://www.lensen-tech.com/. 